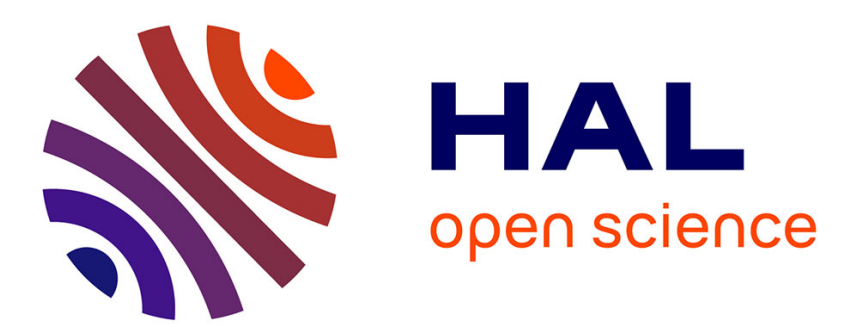

\title{
Opérateurs invariants sur certains immeubles affines de rang 2
}

Ferdaous Kellil, Guy Rousseau

\section{To cite this version:}

Ferdaous Kellil, Guy Rousseau. Opérateurs invariants sur certains immeubles affines de rang 2. Annales de la Faculté des Sciences de Toulouse. Mathématiques., 2007, XVI (3), pp.591-610. 10.5802/afst.1160 . hal-00094818

\section{HAL Id: hal-00094818 https://hal.science/hal-00094818}

Submitted on 15 Sep 2006

HAL is a multi-disciplinary open access archive for the deposit and dissemination of scientific research documents, whether they are published or not. The documents may come from teaching and research institutions in France or abroad, or from public or private research centers.
L'archive ouverte pluridisciplinaire HAL, est destinée au dépôt et à la diffusion de documents scientifiques de niveau recherche, publiés ou non, émanant des établissements d'enseignement et de recherche français ou étrangers, des laboratoires publics ou privés. 


\title{
Opérateurs invariants sur certains immeubles affines de rang 2
}

\author{
Ferdaous Kellil \\ Guy Rousseau
}

\begin{abstract}
Résumé
On considère un immeuble $\Delta$ de type $\widetilde{A_{2}}$ ou $\widetilde{B_{2}}$, différents sousensembles $\mathcal{S}^{\prime}$ de l'ensemble $\mathcal{S}$ des sommets de $\Delta$ et différents groupes $G$ d'automorphismes de $\Delta$, très fortement transitifs sur $\Delta$. On montre que l'algèbre des opérateurs $G$-invariants agissant sur l'espace des fonctions sur $\mathcal{S}^{\prime}$ est souvent non commutative ( contrairement aux résultats classiques ). Dans certains cas on décrit sa structure et on détermine ses fonctions radiales propres. On en déduit que la conjecture d'Helgason n'est pas toujours vérifiée dans ce cadre.
\end{abstract}

\section{Abstract}

We consider a building $\Delta$ of type $\widetilde{A_{2}}$ or $\widetilde{B_{2}}$, different subsets $\mathcal{S}^{\prime}$ of the set $\mathcal{S}$ of vertices in $\Delta$ and different automorphism groups $G$, very strongly transitive on $\Delta$. We prove that the algebra of $G$ invariant operators acting on the space of functions on $\mathcal{S}^{\prime}$ is often not commutative ( contrarily to the classical results). In some cases we describe its structure, determine its radial eigunfunctions and deduce that the Helgason conjecture is not verified in this context.

\section{Introduction}

Si $G$ est un groupe réductif sur un corps local non archimédien et $U$ en est un "bon" sous-groupe compact ouvert alors l'algèbre de convolution $\mathcal{H}(G, U)$, des fonctions complexes bi- $U$-invariantes à support compact sur

Keywords : Buildings, Laplace operators, Poisson kernel, Convolution algebra.

Math. Subject Classification : Primary : 51E24, 22E35. Secondary : 43A85, 39A12. 


\section{Kellil et Rousseau}

$G$ est commutative, comme l'a montré Ichiro Satake [S 63]. Les fonctions $U$-invariantes sur $G$, qui sont fonctions propres de $\mathcal{H}(G, U)$ ont été l'objet de nombreux travaux d'analyse harmonique, en particulier celles qui sont bi- $U$-invariantes sont les fonctions sphériques zonales [S 63], [McD 71].

Mais $G / U$ s'identifie à une partie $\mathcal{S}^{\prime}$ de l'ensemble $\mathcal{S}$ des sommets de l'immeuble de Bruhat-Tits $\Delta$ de $G$. Les fonctions $U$-invariantes sur $G$ sont des fonctions sur $\mathcal{S}^{\prime}$ et $\mathcal{H}(G, U)$ opère sur ces fonctions. On va donc se placer dans un cadre plus abstrait.

Soit $\Delta$ un immeuble épais localement fini et $\mathcal{S}^{\prime}$ un sous ensemble de l'ensemble $\mathcal{S}$ de ses sommets. On suppose qu'il existe un groupe $G$ d'automorphismes de $\Delta$ qui stabilise $\mathcal{S}^{\prime}$ et agit "très fortement transitivement" sur $\Delta$. Un opérateur $K$ est une fonction "localement finie" sur $\mathcal{S}^{\prime} \times \mathcal{S}^{\prime}$. Ces opérateurs forment une algèbre qui agit sur l'espace des fonctions sur $\mathcal{S}^{\prime}$ (cf. 1.1). On s'intéresse à l'algèbre $\mathcal{O}^{\prime}$ des opérateurs $G$-invariants, i.e. vérifiant $K(g u, g v)=K(u, v), \forall g \in G, \forall u, v \in \mathcal{S}^{\prime}$. Si on suppose $G$ transitif sur $\mathcal{S}^{\prime}$ alors un tel opérateur s'identifie à une fonction à support fini sur $G / K_{s}$ où $K_{s}$ est le fixateur dans $G$ d'un sommet $s$ de $\mathcal{S}^{\prime}$.

Ce point de vue a été adopté dans plusieurs articles antérieurs [C 01], [CM 94], [CW 04], [GL 99], [MZ 00] et [MZ 02]. Mais ici on va étudier, apparemment pour la première fois, le cas où l'algèbre $\mathcal{O}^{\prime}$ d'opérateurs est non commutative. Plus précisément on considère un immeuble affine $\Delta$ de type $\widetilde{A_{2}}$ ou $\widetilde{B_{2}}$, différents ensembles $\mathcal{S}^{\prime}$ de sommets et différents groupes $G$ très fortement transitifs sur $\Delta$. On va étudier la commutativité de $\mathcal{O}^{\prime}$, et pour certains cas, élucider précisément sa structure et déterminer ses fonctions radiales propres.

Au premier paragraphe on prend $\Delta$ de type $\widetilde{A_{2}}$ et on étudie l'algèbre $\mathcal{O}_{i}$ des opérateurs sur l'ensemble $\mathcal{S}_{i}$ des sommets de type $i$ invariants par un groupe $G$ ( très fortement transitif ) stabilisant $\mathcal{S}_{i}$, puis l'algèbre $\mathcal{O}_{1,2}$ des opérateurs sur $\mathcal{S}_{1} \cup \mathcal{S}_{2}$, invariants par $G$, stabilisant $\mathcal{S}_{1} \cup \mathcal{S}_{2}$ et transitif sur $\mathcal{S}_{1} \cup \mathcal{S}_{2}$. On détermine entièrement la structure de ces deux algèbres.

Au second paragraphe, on détermine la dimension de l'ensemble des fonctions propres pour $\mathcal{O}_{i}$ puis pour $\mathcal{O}_{1,2}$ qui sont de plus radiales, c'est à dire invariantes par le sous-groupe $K_{s}$ fixateur d'un sommet $s$ de $\mathcal{S}$. Cela nous permet de montrer que la transformation de Poisson qui à une mesure additive finie sur la frontière de $\Delta$ associe une fonction propre de $\mathcal{O}_{i}$ ( ou $\mathcal{O}_{1,2}$ ) ne peut pas toujours être bijective : la conjecture d'Helgason n'est pas vérifiée dans ce cas.

$\mathrm{Au}$ troisième paragraphe, on se place dans le cas où $\Delta$ est de type $\widetilde{B_{2}}$ 
et le groupe $G$ stabilise les types. On détermine entièrement la structure de l'algèbre $\mathcal{O}_{0,2}$ des opérateurs sur $\mathcal{S}_{0} \cup \mathcal{S}_{2}$ puis de l'algèbre $\mathcal{O}_{1}$ des opérateurs sur $\mathcal{S}_{1}$ invariants par un tel groupe.

$\mathrm{Au}$ dernier paragraphe, on compare les résultats obtenus avec les résultats classiques de Satake. On montre en particulier en quoi $K_{s}$ n'est pas toujours un "bon" sous-groupe.

\section{Opérateurs sur un immeuble de type $\widetilde{A_{2}}$,}

\section{$1.1 \quad$ Notations}

Considérons un immeuble épais $\Delta$ de type $\widetilde{A_{2}}$, et d'ordre $q$, comme défini par exemple dans [MZ 00 ], voir aussi [R 89]. On note $\mathcal{S}$ l'ensemble de ses sommets et $\mathcal{C}$ l'ensemble de ses chambres. Désignons par $\tau: \mathcal{S} \rightarrow \mathbb{Z} / 3 \mathbb{Z}$ la fonction type. Pour $i \in \mathbb{Z} / 3 \mathbb{Z}$, on note $\mathcal{S}_{i}$ l'ensemble des sommets de type $i$. Chaque chambre contient un et un seul sommet de chaque type. Un appartement de $\Delta$ peut s'identifier à $\mathbb{R}^{2}$ pavé par des triangles équilatéraux qui sont les chambres de cet appartement. Une cloison est une arête d'une chambre, son type est le type du sommet opposé; elle est contenue dans $q+1$ chambres ( la valence $q$ ne dépend pas du type ). Une galerie de longueur $n$ est une suite $C_{0}, C_{1}, \ldots, C_{n}$ de chambres telles que $C_{i} \cap C_{i-1}$ contienne une cloison, son type est la suite des types de ces $n$ cloisons.

Soit $A$ un appartement, $x$ un sommet de $A$ et $C$ une chambre de $A$ contenant $x$, il existe un unique système de coordonnées sur l'appartement $A$ tel que $x=x_{0,0}, \mathcal{S} \cap C=\left\{x_{0,0}, x_{1,0}, x_{0,1}\right\}$ et si $i=\tau\left(x_{0,0}\right)$ alors $\tau\left(x_{1,0}\right)=i+1$ et $\tau\left(x_{0,1}\right)=i-1$. Alors les sommets de $A$ sont indexés par $\mathbb{Z}^{2}, \tau\left(x_{j, k}\right) \equiv$ $i+j-k$ (modulo 3$)$ et $\left\{x_{j, k} / j, k \in\left[0,+\infty[\}\right.\right.$ est le quartier $Q_{x, C}$ (sector) de $A$ de sommet $x$ contenant $C$.

Pour $j, k \in \mathbb{N}$, on note $V_{j, k}(x)$ l'ensemble des points $x_{j, k}$ associés aux différents choix de $A$ et $C$ tels que $x \in C \subset A$. L'ensemble $\mathcal{S}$ est réunion disjointe des $V_{j, k}(x)$.

Un groupe $G$ d'automorphismes de $\Delta$ est dit "très fortement transitif' si le sous groupe $G_{0}$ de $G$ formé des éléments conservant les types agit transitivement sur les paires $(A, C)$ où $C$ est une chambre de l'appartement $A$. Alors, pour $x \in \mathcal{S}$, les $V_{j, k}(x)$ sont les orbites dans $\mathcal{S}$ du groupe $G_{0 x}$ des éléments de $G_{0}$ fixant $x$.

Un opérateur $K$ est une fonction sur $\mathcal{S} \times \mathcal{S}$. On ne considérera que des 


\section{Kellil et Rousseau}

fonctions localement finies i.e. telles que pour tout $u \in \mathcal{S},\{v \in \mathcal{S} / K(u, v) \neq$ $0\}$ est fini. Le produit des opérateurs $K, K^{\prime}$ est donné par $K * K^{\prime}(s, t)=$ $\sum_{u} K(s, u) K^{\prime}(u, t)$. Un tel opérateur agit sur les fonctions par $(K * f)(s)=$ $\sum_{u} K(s, u) f(u)$. On s'intéresse aux opérateurs (localement finis) invariants par différents groupes très fortement transitifs.

On définit des opérateurs $K_{j, k}^{i} \operatorname{sur} \mathcal{S} \times \mathcal{S}($ pour $i \in \mathbb{Z} / 3 \mathbb{Z}$ et $j, k \in \mathbb{N})$ par :

$$
K_{j, k}^{i}(u, v)=\left\{\begin{array}{ccc}
1 & \text { si } \tau(u)=i \text { et } v \in V_{j, k}(u) \\
0 & \text { sinon }
\end{array}\right.
$$

Le terme dominant de l'opérateur $\sum C_{j, k}^{i} K_{j, k}^{i}$ ( non nul mais avec des constantes $C_{j, k}^{i}$ presque toutes nulles ) est $\sum_{j+k=N} C_{j, k}^{i} K_{j, k}^{i}$ où $N=\max \{j+$ $\left.k \quad / C_{j, k}^{i} \neq 0\right\}$ est le degré total de l'opérateur.

On considère un groupe $G$ très fortement transitif et conservant les types, on montre facilement que l'algèbre $\mathcal{O}$ des opérateurs $G$-invariants admet comme base les $K_{j, k}^{i}(i \in \mathbb{Z} / 3 \mathbb{Z}$ et $j, k \in \mathbb{N})$. On peut définir cette algèbre $\mathcal{O}$ même en l'absence d'un tel groupe $G$.

\section{Proposition 1.1:}

1) $K_{j, k}^{i} K_{m, n}^{l}=0$ si $l \not \equiv i+j-k$ modulo (3).

2) Pour $l \equiv i+j-k$ (modulo 3$)$, le terme dominant de $K_{j, k}^{i} K_{m, n}^{l}$ est $K_{j+m, k+n}^{i}$.

3) L'algèbre $\mathcal{O}$ est engendrée par les opérateurs $K_{0,0}^{i}$, $K_{0,1}^{i}$ et $K_{1,0}^{i}$ pour $i \in \mathbb{Z} / 3 \mathbb{Z}$. Elle n'est pas commutative.

Démonstration : la première assertion est claire. En particulier on a $K_{1,0}^{0} K_{1,0}^{2}=0$ et $K_{1,0}^{2} K_{1,0}^{0} \neq 0$. Par ailleurs, on remarque que, lorsque le produit est non nul, le terme dominant dans $K_{j, k}^{i} K_{0,1}^{l}\left(\operatorname{resp} . K_{j, k}^{i} K_{1,0}^{l}\right)$ est $K_{j, k+1}^{i}\left(\right.$ resp. $\left.K_{j+1, k}^{i}\right)$. Par un raisonnement par récurrence on obtient alors 2) et 3).

Les opérateurs $L_{1}=K_{1,0}^{0}+K_{1,0}^{1}+K_{1,0}^{2}$ et $L_{2}=K_{0,1}^{0}+K_{0,1}^{1}+K_{0,1}^{2}$ sont ( à une constante multiplicative près) les opérateurs de Laplace considérés par Mantero et Zappa [MZ 00], ils commutent donc entre eux. Si $G$ est un groupe très fortement transitif, transitif sur $\mathcal{S}$ et permutant circulairement les types, l'algèbre $\mathcal{O}_{\text {circ }}$ des opérateurs $G$-invariants est engendrée par $L_{1}$ et $L_{2}$; c'est 
une algèbre (commutative) de polynômes, elle est égale à $\mathbb{C}\left[L_{1}, L_{2}\right], c f$. [MZ $00]$.

Si $T$ est un opérateur et si $i, j \in \mathbb{Z} / 3 \mathbb{Z}$, on définit l'opérateur ${ }_{i}|T|_{j}$ par :

$$
{ }_{i}|T|_{j}(u, v)=\left\{\begin{array}{ccc}
T(u, v) & \text { si } \tau(u)=i \text { et } \tau(v)=j \\
0 & \text { sinon }
\end{array}\right.
$$

En particulier ${ }_{i}\left|L_{1}\right|_{j}=K_{1,0}^{i}$ si $j=i+1(0$ sinon $)$ et ${ }_{i}\left|L_{2}\right|_{j}=K_{0,1}^{i}$ si $j=i-1$ (0 sinon).

\subsection{Opérateurs sur $\mathcal{S}_{i}$}

Les opérateurs sur $\mathcal{S}_{i}$ sont considérés comme des opérateurs sur $\mathcal{S}$ nuls en dehors de $\mathcal{S}_{i} \times \mathcal{S}_{i}$. On considère l'algèbre $\mathcal{O}_{i}$ des opérateurs sur $\mathcal{S}_{i}$ invariants par un groupe $G$ très fortement transitif et stabilisant $\mathcal{S}_{i}$; c'est la sous-algèbre de $\mathcal{O}$ de base les $K_{j, k}^{i}$ pour $j \equiv k$ (modulo 3 ). On peut définir cette algèbre $\mathcal{O}_{i}$ même en l'absence d'un tel groupe $G$. On vérifie facilement grâce à la proposition 1.1, qu'elle est engendrée par $K_{0,0}^{i}$ (identité sur $\mathcal{S}_{i}$ ), $K_{1,1}^{i}, K_{3,0}^{i}$ et $K_{0,3}^{i}$. Notons $X_{i}={ }_{i}\left|L_{1} L_{2}\right|_{i}, Y_{i}={ }_{i}\left|L_{1}^{3}\right|_{i}$ et $Z_{i}={ }_{i}\left|L_{2}^{3}\right|_{i}$.

Théorème 1.2: L'algèbre $\mathcal{O}_{i}$ des opérateurs invariants sur $\mathcal{S}_{i}$ est une algèbre commutative : $\mathcal{O}_{i}=\mathbb{C}\left[X_{i}, Y_{i}, Z_{i}\right]$ avec pour seule relation $X_{i}^{3}=Y_{i} Z_{i}$. De plus une base de $\mathcal{O}_{i}$ est constituée des $X_{i}^{m} Y_{i}^{n}$ et $X_{i}^{m} Z_{i}^{n}$ pour $m, n \in \mathbb{N}$.

Démonstration : Comme un sommet de type $i$ a $1+q+q^{2}$ voisins de type $i+1$ (ou $i-1)(c f$. [MZ 00], on voit facilement que

$$
\begin{gathered}
{ }_{i}\left|L_{1} L_{2}\right|_{i}=K_{1,1}^{i}+\left(1+q+q^{2}\right) K_{0,0}^{i} . \\
{ }_{i}\left|L_{1}^{3}\right|_{i}=K_{3,0}^{i}+(2 q+1) K_{1,1}^{i}+(q+1)\left(1+q+q^{2}\right) K_{0,0}^{i} . \\
{ }_{i}\left|L_{2}^{3}\right|_{i}=K_{0,3}^{i}+(2 q+1) K_{1,1}^{i}+(q+1)\left(1+q+q^{2}\right) K_{0,0}^{i} .
\end{gathered}
$$

Comme les opérateurs $L_{1}$ et $L_{2}$ commutent il en est de même pour $X_{i}, Y_{i}$ et $Z_{i}$ et on a $X_{i}^{3}=Y_{i} Z_{i}$. De plus le terme dominant dans $X_{i}^{m} Y_{i}^{p}$ est $K_{m+3 p, m}^{i}$, ainsi les opérateurs $X_{i}$ et $Y_{i}$ sont des variables algébriquement indépendantes et on a les résultats énoncés. 


\section{Kellil et Rousseau}

\subsection{Opérateurs sur $\mathcal{S}_{1} \cup \mathcal{S}_{2}$}

On considère des opérateurs sur $\mathcal{S}_{1} \cup \mathcal{S}_{2}$, donc nuls en dehors de $\left(\mathcal{S}_{1} \cup \mathcal{S}_{2}\right)^{2}$. On note $\mathcal{O}_{1,2}$ l'algèbre de ces opérateurs qui sont invariants par un groupe $G$ très fortement transitif, stabilisant $\mathcal{S}_{1} \cup \mathcal{S}_{2}$ ( donc $\mathcal{S}_{0}$ ) et transitif sur $\mathcal{S}_{1} \cup \mathcal{S}_{2}$.

L'algèbre des opérateurs sur $\mathcal{S}_{1} \cup \mathcal{S}_{2}$ invariants par $G_{0}$ est la sous algèbre de $\mathcal{O}$ de base les $K_{j, k}^{1}$, avec $j \equiv k$ ou $j \equiv k+1$ (modulo 3$)$ et $K_{l, m}^{2}$, avec $l \equiv m$ ou $l \equiv m-1($ modulo 3$)$. Mais $G$ est engendré par $G_{0}$ et un élément $\sigma$ qui stabilise un triplet $x \in C \subset A$ (où $x$ est de type 0 ) et qui échange les sommets de type 1 et 2 de $C$. Alors $\sigma$ transforme $K_{j, k}^{1}$ en $K_{k, j}^{2}$. Donc l'algèbre $\mathcal{O}_{1,2}$ admet pour base les $K_{j, k}^{1}+K_{k, j}^{2}$ pour $j \equiv k$ ou $j \equiv k+1$. On peut ainsi définir cette algèbre même en l'absence du groupe $G$.

On note $X=X_{1}+X_{2}, \quad Y=Y_{1}+Z_{2}$ et $Z=Z_{1}+Y_{2}$.

La sous-algèbre $\mathbb{C}[X, Y, Z]$ de $\mathcal{O}_{1,2}$ est formée d'opérateurs conservant les types. Elle est isomorphe (par restriction) à $\mathbb{C}\left[X_{1}, Y_{1}, Z_{1}\right]=\mathcal{O}_{1}$ ou $\mathbb{C}\left[X_{2}, Y_{2}, Z_{2}\right]=\mathcal{O}_{2}$.

On définit $L=K_{1,0}^{1}+K_{0,1}^{2}$ et $M=(q+1) L+K_{0,2}^{1}+K_{2,0}^{2}$. On a :

$L={ }_{1}\left|L_{1}\right|_{2}+{ }_{2}\left|L_{2}\right|_{1}$ et $M={ }_{2}\left|L_{1}^{2}\right|_{1}+{ }_{1}\left|L_{2}^{2}\right|_{2}$.

Théorème 1.3: $\mathcal{O}_{1,2}$ est une algèbre non commutative engendrée par $L$ et $M$. On a $\mathcal{O}_{1,2}=\mathbb{C}[X, Y, Z] \oplus L \mathbb{C}[X, Y, Z] \oplus\left(\oplus_{p \geq 0} \mathbb{C} M Y^{p}\right)$. La structure multiplicative est donnée par les relations :

$$
\begin{gathered}
L^{2}=X ; L M=Y ; M L=Z ; M^{2}=L^{4}=X^{2} \\
X L=L X ; Y L=L Z ; Z L=M X=L Y
\end{gathered}
$$

et

$$
X M=L Y ; Y M=M Z=L X^{2} ; Z M=M Y
$$

Démonstration : Les dernières relations sont faciles à vérifier, en particulier $X, Y$ et $Z$ s'expriment en fonction de $L$ et $M$. D'après la proposition 1.1 on détermine les termes dominants des monômes suivants en $L, M, X, Y, Z$ :

$K_{n, n+3 p}^{1}+K_{n+3 p, n}^{2}$ pour $X^{n} Y^{p}, K_{n+3 p, n}^{1}+K_{n, n+3 p}^{2}$ pour $X^{n} Z^{p}$

$K_{0,3 p+2}^{1}+K_{3 p+2,0}^{2}$ pour $M Y^{p}, K_{n+1, n+3 p}^{1}+K_{n+3 p, n+1}^{2}$ pour $L X^{n} Y^{p}$ et $K_{n+3 p+1, n}^{1}+K_{n, n+3 p+1}^{2}$ pour $L X^{n} Z^{p}$.

Ainsi ces monômes forment une base de $\mathcal{O}_{1,2}$ et le théorème est démontré. $\diamond$ 


\section{Fonctions propres radiales pour $\mathcal{O}_{i}$ et $\mathcal{O}_{1,2}$}

\subsection{Noyau de Poisson et résultats de Mantero et Zappa}

On considère tous les quartiers de tous les appartements de l'immeuble et la relation d'équivalence sur ces quartiers définie par $Q \sim Q^{\prime} \Leftrightarrow Q \cap Q^{\prime}$ contient un sous-quartier. L'ensemble quotient $\Omega$ est la frontière de l'immeuble $c f$. [MZ 00].

Notons $H(\Omega)$ l'espace des fonctions localement constantes sur $\Omega$, son espace dual $H^{\prime}(\Omega)$ est l'ensemble des mesures additives finies sur $\Omega$, ( $c f$. [MZ 00] ).

On choisit un sommet $x_{0}$, une chambre $C$ et un appartement $A$ tels que $x_{0} \in C \subset A$. On note $\omega$ la classe d'équivalence du quartier $Q_{x_{0}, C}$ associé. Le sous-groupe des éléments de $G$ qui fixent (point par point) un sous-quartier (quelconque) de $Q_{x_{0}, C}$ est noté $G_{\omega}$. Si $\alpha=\left(a_{1}, a_{2}, a_{3}\right) \in\left(\mathbb{C}^{*}\right)^{3}$ vérifie $a_{1} a_{2} a_{3}=1$, Mantero et Zappa construisent (par des rétractions) une fonction $P_{\alpha}^{x_{0}}(-, \omega)$ sur $\mathcal{S}$ telle que $P_{\alpha}^{x_{0}}\left(x_{m, n}, \omega\right)=a_{1}^{m} a_{3}^{-n}$ et telle que $P_{\alpha}^{x_{0}}$ est invariante par $G_{\omega}$. Cette fonction est propre pour $L_{1}$ et $L_{2}: L_{1} P_{\alpha}^{x_{0}}=$ $\gamma_{1}(\alpha) P_{\alpha}^{x_{0}}, L_{2} P_{\alpha}^{x_{0}}=\gamma_{2}(\alpha) P_{\alpha}^{x_{0}}$ avec $\gamma_{1}(\alpha)=a_{1}+q a_{2}+q^{2} a_{3}$ et $\gamma_{2}(\alpha)=$ $a_{3}^{-1}+q a_{2}^{-1}+q^{2} a_{1}^{-1}$ et tout couple $\left(\gamma_{1}, \gamma_{2}\right) \in \mathbb{C}^{2}$ est ainsi obtenu, [MZ 00].

On va considérer ici des fonctions propres pour certains opérateurs qui sont de plus invariantes par le sous-groupe $K_{s}$ de $G$ fixateur d'un sommet $s$ (et non plus par $G_{\omega}$ ). Ces fonctions sont précisément celles qui sont constantes sur les ensembles $V_{j, k}(s)$, on peut aussi les qualifier de $s$-radiales; ceci permet de les définir même en l'absence d'un groupe $G$ très fortement transitif.

\subsection{Fonctions propres radiales pour $\mathcal{O}_{i}$}

On note $\mathcal{F}_{s}^{i}\left(x_{i}, y_{i}, z_{i}\right)$ l'ensemble des fonctions $K_{s}$-invariantes sur $\mathcal{S}_{i}$, qui sont propres pour $\mathcal{O}_{i}$ : plus précisément $X_{i} f=x_{i} f, Y_{i} f=y_{i} f$ et $Z_{i} f=z_{i} f$ pour $\left(x_{i}, y_{i}, z_{i}\right) \in \mathbb{C}^{3}$ vérifiant $x_{i}^{3}=y_{i} z_{i}$.

\section{Remarques 2.1:}

1)Soit $f$ une fonction sur $\mathcal{S}$ invariante par $K_{s}$ et propre de $L_{1}$ (respectivement de $\left.L_{2}\right)$ pour la valeur propre $\gamma_{1}\left(\right.$ resp. $\left.\gamma_{2}\right)$. Alors $f_{\mathcal{S}_{i}}$ est propre pour $X_{i}, Y_{i}$ et $Z_{i}$ pour les valeurs propres respectives $\gamma_{1} \gamma_{2}, \gamma_{1}^{3}, \gamma_{2}^{3}$; (car $\left.X_{i}={ }_{i}\left|L_{1} L_{2}\right|{ }_{i}, \quad Y_{i}={ }_{i}\left|L_{1}^{3}\right|_{i}, \quad Z_{i}={ }_{i}\left|L_{2}^{3}\right|_{i}\right)$. 


\section{Kellil et Rousseau}

2)On veut trouver les conditions sur $\gamma_{1}, \gamma_{2}$ pour que $f_{\left.\right|_{\mathcal{S}_{i}}} \in \mathcal{F}_{s}^{i}\left(x_{i}, y_{i}, z_{i}\right)$. Cela revient à résoudre $x_{i}=\gamma_{1} \gamma_{2}, \quad y_{i}=\gamma_{1}^{3}, \quad z_{i}=\gamma_{2}^{3}$.

Pour $y_{i} \neq 0$ ou $z_{i} \neq 0$; en résolvant $y_{i}=\gamma_{1}^{3}$ on a 3 choix pour $\gamma_{1}$ et 3 choix pour $\gamma_{2}$ en résolvant $z_{i}=\gamma_{2}^{3}$. Et comme $x_{i}=\gamma_{1} \gamma_{2}$ on a alors 3 choix pour $\gamma_{1}, \gamma_{2}$ vérifiant les trois équations en même temps et qui correspondent à $\left(\gamma_{1}, \gamma_{2}\right)$ ou $\left(\zeta \gamma_{1}, \bar{\zeta} \gamma_{2}\right)$ ou encore $\left(\bar{\zeta} \gamma_{1}, \zeta \gamma_{2}\right),\left(\right.$ avec $\left.\zeta=\frac{-1}{2}+i \frac{\sqrt{3}}{2}\right)$.

Pour $y_{i}=0$ et $z_{i}=0$ on a $x_{i}=0$ et $\gamma_{1}=\gamma_{2}=0$.

\section{Proposition 2.2:}

$\operatorname{dim} \mathcal{F}_{s}^{i}\left(x_{i}, y_{i}, z_{i}\right) \leq 1$ si $s \in \mathcal{S}_{i}$

$\operatorname{dim} \mathcal{F}_{s}^{i}\left(x_{i}, y_{i}, z_{i}\right) \leq 2$ si $s \in \mathcal{S}_{j}, j \neq i$

\section{Démonstration :}

Soit $f^{s} \in \mathcal{F}_{s}^{i}\left(x_{i}, y_{i}, z_{i}\right)$ alors $f^{s}$ est constante $\operatorname{sur} V_{j, k}(s)$. Notons $f^{s}(u)=$ $f_{j, k}^{s}$ si $u \in V_{j, k}(s)$. Par un raisonnement par récurrence sur $n=j+k$ et en utilisant les équations $X_{i} f^{s}(u)=x_{i} f^{s}(u), Y_{i} f^{s}(u)=y_{i} f^{s}(u)$ et $Z_{i} f^{s}(u)=$ $z_{i} f^{s}(u)$, on montre que les $f_{j, k}^{s}$ se déterminent d'une façon linéaire en fonction de :

$$
\left\{\begin{array}{ccccc}
f_{0,0}^{s} & \text { si } & s \in \mathcal{S}_{i} \\
f_{0,1}^{s} & \text { et } & f_{2,0}^{s} & \text { si } & s \in \mathcal{S}_{i+1} \\
f_{1,0}^{s} & \text { et } & f_{0,2}^{s} & \text { si } & s \in \mathcal{S}_{i-1}
\end{array}\right.
$$

\section{Proposition 2.3:}

$\operatorname{dim} \mathcal{F}_{s}^{i}\left(x_{i}, y_{i}, z_{i}\right) \geq 1$ si $s \in \mathcal{S}_{i}$ ou $s \in \mathcal{S}_{j}, j \neq i$ et $\left(y_{i} \neq 0\right.$ ou $\left.z_{i} \neq 0\right)$ $\operatorname{dim} \mathcal{F}_{s}^{i}\left(x_{i}, y_{i}, z_{i}\right) \geq 2$ si $s \in \mathcal{S}_{j}, j \neq i$ et $\left(x_{i}=y_{i}=z_{i}=0\right)$.

\section{Démonstration :}

D'après la remarque ci-dessus et les résultats rappelés dans la sous-section 2.1, il existe $\alpha$ tel que $P_{\alpha}^{s}$ soit propre pour $X_{i}, Y_{i}, Z_{i}, L_{1}$ et $L_{2}$ avec les valeurs propres $x_{i}, y_{i}, z_{i}, \gamma_{1}$ et $\gamma_{2}$ ( si on fixe $\omega$ comme en 2.1 ). Notons $P_{i}=\left.P_{\alpha}^{s}(., \omega)\right|_{S_{i}}$ et $f_{i}^{s}(t)=\int_{K_{s}} P_{i}(k t, \omega) d k$. Ainsi la fonction $f_{i}^{s}$ est propre pour $X_{i}, Y_{i}, Z_{i}$ avec les mêmes valeurs propres, elle est de plus $K_{s}$-invariante. Notons $j=$ $\tau(s)$.

1) Si $j=i, P_{\alpha}^{s}(s, \omega)=1$ donc $f_{i}^{s}(s)=1 \neq 0$. Ainsi $\operatorname{dim}_{s}^{i}\left(x_{i}, y_{i}, z_{i}\right) \geq 1$.

2)Supposons que $j \neq i$ et (pour fixer les idées) $i=1$. Notons $s_{1} \in \mathcal{S}_{1}$ un sommet à distance 1 de $s$ et $s_{1}^{\prime}$ un sommet de $\mathcal{S}_{1}$ tel que le type de la 
galerie minimale tendue de $s$ à $s_{1}^{\prime}$ soit $(j, 1)$. On suppose $s_{1}$ et $s_{1}^{\prime}$ dans le même quartier de sommet $s$.

Supposons $j=2$. On a ${ }_{2}\left|L_{2}\right|_{1}=K_{0,1}^{2},{ }_{2}\left|L_{1}^{2}\right|_{1}=K_{2,0}^{2}+(q+1)_{2}\left|L_{2}\right|_{1}$ et $P_{\alpha}^{s}(s, \omega)=1$. Par suite $f_{1}^{s}\left(s_{1}\right)=\frac{\gamma_{2}}{1+q+q^{2}}$ et $f_{1}^{s}\left(s_{1}^{\prime}\right)=\frac{\gamma_{1}^{2}-(q+1) \gamma_{2}}{q^{2}\left(1+q+q^{2}\right)}$.

Si $j=0$ les résultats sont les mêmes en échangeant $\gamma_{1}$ et $\gamma_{2}$.

Pour $y_{i} \neq 0$ ou $z_{i} \neq 0$, d'après la remarque ci-dessus $\gamma_{1}$ ou $\gamma_{2}$ n'est pas nul, donc $f_{1}^{s}$ n'est pas nul et la dimension est donc bien supérieure ou égale à 1 .

3)Supposons toujours $j \neq i=1$ et $j=2$. Si $\gamma_{2} \in \mathbb{C}^{*}$ (resp. $\gamma_{1} \in$ $\left.\mathbb{C}^{*}\right)$, on note $g_{0, \gamma_{2}}=\frac{1}{\gamma_{2}} P_{1}$ (resp. $\left.g_{\gamma_{1}, 0}=\frac{1}{\gamma_{1}^{2}} P_{1}\right)$ où $P_{1}$ est définie comme cidessus pour $\gamma_{1}=0$ et $\gamma_{2}$ (resp. $\gamma_{2}=0$ et $\gamma_{1}$ ). En moyennant par $K_{s}$ on obtient des fonctions $f_{0, \gamma_{2}}$ et $f_{\gamma_{1}, 0}$ telles que $f_{0, \gamma_{2}}\left(s_{1}\right)=\frac{1}{1+q+q^{2}}, f_{0, \gamma_{2}}\left(s_{1}^{\prime}\right)=$ $\frac{-(q+1)}{q^{2}\left(1+q+q^{2}\right)}, f_{\gamma_{1}, 0}\left(s_{1}\right)=0$ et $f_{\gamma_{1}, 0}\left(s_{1}^{\prime}\right)=\frac{1}{q^{2}\left(1+q+q^{2}\right)}$. D'après la démonstration de la proposition 2.2 une fonction $K_{s}$-invariante propre pour $X_{1}, Y_{1}$ et $Z_{1}$ est déterminée de façon polynômiale en fonction de ses valeurs en $s_{1}$ et $s_{1}^{\prime}$ et des valeurs propres $x_{1}, y_{1}$ et $z_{1}$. En passant à la limite pour $\gamma_{2} \rightarrow 0$ et $\gamma_{1} \rightarrow 0$ on obtient deux fonctions $f_{0, *}$ et $f_{*, 0} \quad K_{s}$-invariantes, propres pour $X_{1}, Y_{1}$ et $Z_{1}$ et linéairement indépendantes. La dimension est donc bien supérieure ou égale à 2 .

Le cas $j=0$ se traite de la même manière.

\section{Théorème 2.4:}

$\operatorname{dim} \mathcal{F}_{s}^{i}\left(x_{i}, y_{i}, z_{i}\right)=1$ si $s \in \mathcal{S}_{i}$ ou $s \in \mathcal{S}_{j}, j \neq i$ et $\left(y_{i} \neq 0\right.$ ou $\left.z_{i} \neq 0\right)$ $\operatorname{dim} \mathcal{F}_{s}^{i}\left(x_{i}, y_{i}, z_{i}\right)=2$ si $s \in \mathcal{S}_{j}, j \neq i$ et $\left(x_{i}=y_{i}=z_{i}=0\right)$.

\section{Démonstration :}

En utilisant la proposition 2.2 et la proposition 2.3, on obtient le résultat si $s \in \mathcal{S}_{i}$ ou si $s \in \mathcal{S}_{j}, j \neq i$ et $\left(x_{i}=y_{i}=z_{i}=0\right)$.

Soit $s \in S_{j}, j \neq i$ et (pour fixer les idées) $i=1$.

Supposons $j=2$. Avec les notations de la proposition 2.2, pour $u \in$ $V_{0,1}(s), v \in V_{2,0}(s)$ et $w \in V_{1,2}(s)$, on a :

(1) $X_{1} f^{s}(u)=x_{1} f_{0,1}^{s}=(q+1) q^{3} f_{1,2}^{s}+(q+1) q^{2} f_{2,0}^{s}+\left(1+2 q+2 q^{2}\right) f_{0,1}^{s}$.

(2) $Y_{1} f^{s}(u)=y_{1} f_{0,1}^{s}=(q+1) q^{5} f_{3,1}^{s}+q^{4} f_{2,0}^{s}+(2 q+1) x_{1} f_{0,1}^{s}-q\left(1+q+q^{2}\right) f_{0,1}^{s}$.

(3) $Z_{1} f^{s}(u)=z_{1} f_{0,1}^{s}=q^{6} f_{0,4}^{s}+(q+1) q^{3}(3 q+1) f_{1,2}^{s}+(2 q+1)(q+1) q^{2} f_{2,0}^{s}+$ $+(q+1)\left(1+2 q+3 q^{2}\right) f_{0,1}^{s}$.

(4) $X_{1} f^{s}(v)=x_{1} f_{2,0}^{s}=(q+1) q^{3} f_{3,1}^{s}+(q+1) q^{2} f_{1,2}^{s}+(2 q+1) q f_{2,0}^{s}+(1+q) f_{0,1}^{s}$. 


\section{Kellil et Rousseau}

(5) $Z_{1} f^{s}(v)=z_{1} f_{2,0}^{s}=(q+1) q^{5} f_{2,3}^{s}+(q+1)(2 q+1) q^{3} f_{3,1}^{s}+3 q^{3}(q+1) f_{1,2}^{s}+$ $+3 q^{2}(q+1) f_{2,0}^{s}+\left(1+3 q+3 q^{2}\right) f_{0,1}^{s}$.

(6) $X_{1} f^{s}(w)=x_{1} f_{1,2}^{s}=q^{3} f_{0,4}^{s}+q^{4} f_{2,3}^{s}+q^{3} f_{3,1}^{s}+q(3 q+1) f_{1,2}^{s}+q f_{2,0}^{s}+f_{0,1}^{s}$.

Par un simple calcul on obtient :

$$
\left(1^{\prime}\right) \quad f_{0,1}^{s}\left[y_{1}-(q+1) x_{1}\right]=x_{1} q^{2} f_{2,0}^{s}
$$

( à l'équation (2) ajouter $q$ fois l'équation (1) pour obtenir $q^{2}(4)$.)

$\left(2^{\prime}\right) f_{0,1}^{s}\left[(q+1) z_{1}-\left(2 q^{2}+2 q+1\right) x_{1}\right]+q^{2} f_{2,0}^{s}\left[z_{1}-(q+1) x_{1}\right]=(q+1) q^{3} x_{1} f_{1,2}^{s}$.

$\left(\operatorname{car}(q+1) \cdot(3)-q^{3}(q+1) \cdot(6)+q^{2} \cdot(5)-q^{2}(q+1) .(4)\right.$ donne $\left.\left(1+2 q+2 q^{2}\right) .(1)\right)$. On voit alors que si :

1) $x_{1} \neq 0$ et comme $x_{1}=y_{1} z_{1}, y_{1} \neq 0$ et $z_{1} \neq 0$. Alors $f_{2,0}^{s}$ se détermine d'une façon unique en fonction de $f_{0,1}^{s}$. On applique la proposition 2.2 et on obtient $\operatorname{dim} \mathcal{F}_{s}^{1}\left(x_{1}, y_{1}, z_{1}\right) \leq 1$.

2) $\quad x_{1}=0, y_{1} \neq 0$ alors $f_{0,1}^{s}=0$ et $\operatorname{dim} \mathcal{F}_{s}^{1}\left(0, y_{1}, 0\right) \leq 1$.

3) $x_{1}=0, z_{1} \neq 0$ alors $f_{2,0}^{s}=-\frac{q+1}{q^{2}} f_{0,1}^{s}$ et $\operatorname{dim} \mathcal{F}_{s}^{1}\left(0,0, z_{1}\right) \leq 1$.

On applique alors la proposition 2.3, on obtient le résultat.

Le cas $j=0$ s'obtient par un raisonnement analogue.

\subsection{Fonctions propres radiales pour $\mathcal{O}_{1,2}$}

Pour $s \in \mathcal{S}$, on veut déterminer les fonctions invariantes par $K_{s}$ propres pour les opérateurs de $\mathcal{O}_{1,2}$ opérant sur $\mathcal{S}_{1} \cup \mathcal{S}_{2}$.

\section{Remarques 2.5:}

1) Si $f$ est propre pour $L$ et $M$ alors elle est propre pour les opérateurs de $\mathcal{O}_{1,2}$. D'autre part comme $L^{4}=M^{2}$, pour qu'une fonction invariante par $K_{s}$ soit propre pour $L$ et $M$ pour respectivement les valeurs propres $\lambda$ et $\mu$ il faut que $\lambda^{4}=\mu^{2}$.

2) Pour $f$ une fonction sur $\mathcal{S}$, invariante par $K_{s}$ et propre pour $L_{1}\left(\right.$ resp. $\left.L_{2}\right)$ pour les valeurs propres $\gamma_{1}\left(\right.$ resp. $\left.\gamma_{2}\right)$, notons $f_{\left.\right|_{\mathcal{S}_{1}}}=f_{1}$ et $f_{\mathcal{S}_{2}}=f_{2}$. On a alors :

$$
\begin{aligned}
& L\left(f_{\left.\right|_{\mathcal{S}_{1}}}\right)=\gamma_{2} f_{\left.\right|_{\mathcal{S}_{2}}}=\gamma_{2} f_{2} \quad, \quad L\left(f_{\left.\right|_{\mathcal{S}_{2}}}\right)=\gamma_{1} f_{\left.\right|_{\mathcal{S}_{1}}}=\gamma_{1} f_{1} \\
& M\left(f_{\left.\right|_{\mathcal{S}_{1}}}\right)=\gamma_{1}^{2} f_{\left.\right|_{\mathcal{S}_{2}}}=\gamma_{1}^{2} f_{2} \quad, \quad M\left(f_{\left.\right|_{\mathcal{S}_{2}}}\right)=\gamma_{2}^{2} f_{\left.\right|_{\mathcal{S}_{1}}}=\gamma_{2}^{2} f_{1} .
\end{aligned}
$$


On veut déterminer les conditions sur $\gamma_{1}, \gamma_{2}\left(\gamma_{1}, \gamma_{2} \neq 0\right)$ pour que $\sqrt{\gamma_{1}} f_{1}+$ $\epsilon \sqrt{\gamma_{2}} f_{2}$ où $\epsilon= \pm 1$ soit propre pour $L$ et $M$. On a :

$$
\begin{aligned}
& L\left(\sqrt{\gamma_{1}} f_{1}+\epsilon \sqrt{\gamma_{2}} f_{2}\right)=\gamma_{2} \sqrt{\gamma_{1}} f_{2}+\epsilon \gamma_{1} \sqrt{\gamma_{2}} f_{1}=\epsilon \sqrt{\gamma_{2}} \sqrt{\gamma_{1}}\left[\sqrt{\gamma_{1}} f_{1}+\epsilon \sqrt{\gamma_{2}} f_{2}\right] . \\
& M\left(\sqrt{\gamma_{1}} f_{1}+\epsilon \sqrt{\gamma_{2}} f_{2}\right)=\gamma_{1}^{2} \sqrt{\gamma_{1}} f_{2}+\epsilon \gamma_{2}^{2} \sqrt{\gamma_{2}} f_{1} .
\end{aligned}
$$

La fonction $\sqrt{\gamma_{1}} f_{1}+\epsilon \sqrt{\gamma_{2}} f_{2}$ est donc propre pour $L$, elle l'est pour $M$ (avec valeur propre $\frac{\epsilon \gamma_{2}^{2} \sqrt{\gamma_{2}}}{\sqrt{\gamma_{1}}}$ ) si et seulement si $\frac{\gamma_{2}^{3}}{\sqrt{\gamma_{1}}}=\gamma_{1}^{2} \sqrt{\gamma_{1}}$ donc $\gamma_{2}^{3}=\gamma_{1}^{3}$, c'est à dire $\gamma_{2} \sqrt{\gamma_{2}}=\eta \gamma_{1} \sqrt{\gamma_{1}}$ où $\eta= \pm 1$.

Notons $\lambda=\epsilon \sqrt{\gamma_{2}} \sqrt{\gamma_{1}}$ valeur propre pour $L$ et $\quad \mu=\epsilon \frac{\gamma_{2}^{2} \sqrt{\gamma_{2}}}{\sqrt{\gamma_{1}}}=\epsilon \frac{\gamma_{2}^{3}}{\sqrt{\gamma_{2}} \sqrt{\gamma_{1}}}=\frac{\gamma_{2}^{3}}{\lambda}$ valeur propre pour $M$. On a en particulier $\gamma_{1}^{3}=\gamma_{2}^{3}=\lambda \mu$.

Pour $\lambda, \mu$ donnés, on a six choix pour $\sqrt{\gamma_{1}}$ tel que $\gamma_{1}^{3}=\lambda \mu$. Pour chaque choix de $\sqrt{\gamma_{1}}$ on a un choix de $\epsilon \sqrt{\gamma_{2}}$ tel que $\lambda=\epsilon \sqrt{\gamma_{1}} \sqrt{\gamma_{2}}$. On vérifie facilement que pour $\epsilon \sqrt{\gamma_{2}}=\frac{\lambda}{\sqrt{\gamma_{1}}}$ et $\gamma_{1}$ tel que $\gamma_{1}^{3}=\lambda \mu$ on a bien $\gamma_{2}^{3}=\lambda \mu$. On a alors six fonctions possibles. Cependant parmi ces choix il y a ceux qui à un signe près donnent la même chose. Plus précisément si on note ${ }_{o} \sqrt{\gamma_{1}}$ une racine sixième de $\gamma_{1}^{3}$ on obtient à un signe près trois fonctions qui correspondent à :

$$
o \sqrt{\gamma_{1}} f_{1}+\frac{\lambda}{{ }_{o} \sqrt{\gamma_{1}}} f_{2} \quad, \quad \zeta_{o} \sqrt{\gamma_{1}} f_{1}+\bar{\zeta} \frac{\lambda}{{ }_{o} \sqrt{\gamma_{1}}} f_{2} \text { et } \bar{\zeta}_{o} \sqrt{\gamma_{1}} f_{1}+\zeta \frac{\lambda}{o \sqrt{\gamma_{1}}} f_{2}
$$

Notons $\mathcal{F}_{s}(\lambda, \mu)$ l'ensemble des fonctions sur $\mathcal{S}_{1} \cup S_{2}, K_{s}$-invariantes et qui sont propres pour $L$ et $M$ pour respectivement les valeurs propres $\lambda$ et $\mu$.

Proposition 2.6: Pour $\lambda$ et $\mu \neq 0$

1) $\operatorname{dim} \mathcal{F}_{s}(\lambda, \mu) \leq 1$ si $s \in \mathcal{S}_{1}$ ou $s \in \mathcal{S}_{2}$.

2) $\operatorname{dim} \mathcal{F}_{s}(\lambda, \mu) \leq 2$ si $s \in \mathcal{S}_{0}$.

\section{Démonstration :}

$f_{s} \in \mathcal{F}_{s}(\lambda, \mu)$ alors $f_{s}$ est constante sur $V_{j, k}(s)$. Notons alors :

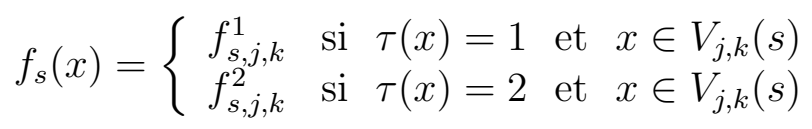

Pour $\lambda$ et $\mu \neq 0$, en écrivant les équations $L f_{s}(x)=\lambda f_{s}(x)$ et $M f_{s}(x)=$ $\mu f_{s}(x)$ pour $x \in V_{j, k}(s)$ et en faisant un raisonnement par récurrence sur $n=j+k$, les $f_{s, j, k}^{1}, f_{s, j, k}^{2}$ se déterminent d'une façon linéaire en fonction de $f_{s, 0,0}^{1}$ (resp. $\left.f_{s, 0,0}^{2}\right)$ si $s \in \mathcal{S}_{1}$ (resp. $s \in \mathcal{S}_{2}$ ) et en fonction de $f_{s, 1,0}^{1}$ et de $f_{s, 0,1}^{2}$ si $s \in \mathcal{S}_{0}$. Ainsi pour $\lambda$ et $\mu \neq 0$, on obtient le résultat. 


\section{Kellil et Rousseau}

Proposition 2.7: Pour $\lambda$ et $\mu \neq 0$

1) $\operatorname{dim} \mathcal{F}_{s}(\lambda, \mu) \geq 1$ si $s \in \mathcal{S}_{1}$ ou $s \in \mathcal{S}_{2}$.

2) $\operatorname{dim} \mathcal{F}_{s}(\lambda, \mu) \geq 2$ si $s \in \mathcal{S}_{0}$.

\section{Démonstration :}

1) Pour $s=s_{1} \in \mathcal{S}_{1}$ ou $s=s_{2} \in \mathcal{S}_{2}$, d'après la remarque 2.5.2 et la sous-section 2.1 ci-dessus il existe $\alpha$ tel que si on note $\left.P_{\alpha}^{s}(-, \omega)\right|_{\mathcal{S}_{1}}=P_{1}$ et $\left.P_{\alpha}^{s}(-, \omega)\right|_{\mathcal{S}_{2}}=P_{2}$ on a :

$L P_{1}=\gamma_{2} P_{2} \quad, \quad L P_{2}=\gamma_{1} P_{1}, \quad M P_{1}=\gamma_{1}^{2} P_{2}$ et $M P_{2}=\gamma_{2}^{2} P_{1}$

et par suite pour $\gamma_{1}$ et $\gamma_{2} \neq 0$ (c'est à dire $\lambda$ et $\mu \neq 0$ ) on obtient une fonction $g_{\eta, 1}\left(\right.$ resp. $\left.g_{\eta, 2}\right)$ pour $s \in \mathcal{S}_{1}\left(\right.$ resp. $s \in \mathcal{S}_{2}$ ) propre pour $L$ et $M$ ( avec $\lambda$ et $\mu$ comme valeurs propres ) et invariante par le fixateur d'un secteur :

$$
g_{\eta, 1}=\frac{1}{\sqrt{\gamma_{1}}}\left[\sqrt{\gamma_{1}} P_{1}+\epsilon \sqrt{\gamma_{2}} P_{2}\right] \quad\left(\text { resp. } \quad g_{\eta, 2}=\frac{1}{\epsilon \sqrt{\gamma_{2}}}\left[\sqrt{\gamma_{1}} P_{1}+\epsilon \sqrt{\gamma_{2}} P_{2}\right]\right)
$$

En moyennant par le groupe compact $K_{s}$, on obtient une fonction invariante par $K_{s}, f_{\eta, 1}\left(\right.$ resp. $\left.f_{\eta, 2}\right)$ lorsque $s \in \mathcal{S}_{1}$ (resp. $s \in \mathcal{S}_{2}$ ). De plus comme $f_{\eta, 1}\left(s_{1}\right)=1 \neq 0$ (resp. $f_{\eta, 2}\left(s_{2}\right)=1 \neq 0$ ) on obtient pour $\lambda$ et $\mu \neq 0$, dim $\mathcal{F}_{s}(\lambda, \mu) \geq 1$ si $s \in \mathcal{S}_{1}$ (resp. $\left.s \in \mathcal{S}_{2}\right)$.

2) Pour $s=s_{0} \in \mathcal{S}_{0}$, considérons pour $\gamma_{1} \neq 0$ et $\gamma_{2} \neq 0, g_{\eta, 0}=$ $\frac{1}{\sqrt{\gamma_{1}} \gamma_{1}}\left[\sqrt{\gamma_{1}} P_{1}+\epsilon \sqrt{\gamma_{2}} P_{2}\right]$ et notons $s_{1} \in \mathcal{S}_{1}, s_{2} \in \mathcal{S}_{2}$ deux sommets contenus dans une même chambre que $s_{0}$. On a $P_{\alpha}^{s}(s, \omega)=1, L_{1} P_{\alpha}^{s}=\gamma_{1} P_{\alpha}^{s}$ et $L_{2} P_{\alpha}^{s}=\gamma_{2} P_{\alpha}^{s}$. En moyennant les fonctions $g_{\eta, 0}$ par $K_{s}$, on obtient deux fonctions $f_{1,0}, f_{-1,0}$ invariantes par $K_{s}$ et propres pour $L$ et $M$ vérifiant :

$$
\begin{aligned}
f_{1,0}\left(s_{1}\right) & =\frac{1}{1+q+q^{2}} \quad, \quad f_{1,0}\left(s_{2}\right)=\frac{1}{1+q+q^{2}} \\
f_{-1,0}\left(s_{1}\right) & =\frac{1}{1+q+q^{2}} \quad, \quad f_{-1,0}\left(s_{2}\right)=\frac{-1}{1+q+q^{2}} .
\end{aligned}
$$

Et comme $f_{1,0}$ et $f_{-1,0}$ sont non nulles et linéairement indépendantes on a alors $\operatorname{dim} \mathcal{F}_{s}(\lambda, \mu) \geq 2$ si $s \in \mathcal{S}_{0}$.

\section{Théorème 2.8:}

$\operatorname{dim} \mathcal{F}_{s}(\lambda, \mu)=1$ si $s \in \mathcal{S}_{1}$ ou $s \in \mathcal{S}_{2}$.

$\operatorname{dim} \mathcal{F}_{s}(\lambda, \mu)=2$ si $s \in \mathcal{S}_{0}$.

\section{Démonstration :}


Pour $\lambda$ et $\mu \neq 0$ il suffit d'appliquer les propositions 2.6 et 2.7. Par ailleurs d'après la proposition 2.6 une fonction invariante par $K_{s}$ et propre pour $L$ et $M$ est déterminée d'une façon polynômiale en fonction des valeurs propres $\lambda, \mu$ et de sa valeur en $s$ ( $\operatorname{si} s \in \mathcal{S}_{1}$ ou si $s \in \mathcal{S}_{2}$ ) ou de ses valeurs en $s_{1}, s_{2}$ tels que $d\left(s, s_{1}\right)=d\left(s, s_{2}\right)=1$ ( si $\left.s \in \mathcal{S}_{0}\right)$. Par prolongement par continuité lorsqu'on fait tendre $\lambda, \mu$ vers 0 le résultat ci-dessus reste alors valable.

\subsection{Transformation de Poisson}

Pour $s \in \mathcal{S}$, on définit la transformation de Poisson $\mathcal{P}_{\alpha}^{s}$ associée à $\alpha \in$ $\left(\mathbb{C}^{*}\right)^{3}(c f .2 .1)$ : elle transforme $\mu \in H^{\prime}(\Omega)$ en $\mathcal{P}_{\alpha}^{s} \mu(x)=\int_{\Omega} P_{\alpha}^{s}(x, \omega) d \mu(\omega), x \in$ $\mathcal{S}$.

Dans le cas où l'ensemble des fonctions propres de $\mathcal{O}_{i}$ ou de $\mathcal{O}_{1,2}$ qui sont invariantes par le fixateur $K_{s}$ d'un sommet fixé dans $\mathcal{S}$ est de dimension deux, la transformation de Poisson $\mathcal{P}_{\alpha}^{s}$ ne peut être bijective.

En effet si $f=\mathcal{P}_{\alpha}^{s} \mu$ est invariante par $K_{s}$, on a $f(x)=\int_{\Omega} P_{\alpha}^{s}(x, \omega) d \mu(\omega)$ et, pour tout $k \in K_{s}$, on a :

$$
\begin{aligned}
f(k x)=f(x) & =\int_{\Omega} P_{\alpha}^{s}(k x, \omega) d \mu(\omega)=\int_{\Omega} P_{\alpha}^{s}\left(x, k^{-1} \omega\right) d \mu(\omega)= \\
& =\int_{\Omega} P_{\alpha}^{s}(x, \omega) d \mu_{k}(\omega), \text { où } \mu_{k}=\left(k^{-1}\right)^{*} \mu .
\end{aligned}
$$

Si $\mathcal{P}_{\alpha}^{s}$ est injective alors $\mu=\left(k^{-1}\right)^{*} \mu, \forall k \in K_{s}$ : la mesure $\mu$ est invariante par $K_{s}$. Mais comme $G$ est très fortement transitif et $s$ est un sommet spécial, le groupe $K_{s}$ est transitif sur $\Omega$, et donc il n'y a qu'une seule mesure $K_{s^{-}}$ invariante sur $\Omega$ à une constante près. L'image de $\mathcal{P}_{\alpha}^{s}$ est de dimension 1 et dans le cas qui nous interesse $\mathcal{P}_{\alpha}^{s}$ ne peut être surjective.

\section{Opérateurs sur un immeuble de type $\widetilde{B_{2}}$,}

Soit $\Delta$ un immeuble de type $\widetilde{B_{2}}$, , comme défini par exemple dans [MZ 02 ]. On peut reprendre les définitions du premier alinea de 1.1. Les chambres sont maintenant des triangles rectangles isocèles et, par convention, l'hypothénuse a pour type 1 , les deux autres côtés ont pour type 0 et 2 . La valence $q_{i}$ d'une cloison dépend de son type $i$; on note $p=q_{0}, q=q_{1}$ et $r=q_{2}$. 


\section{Kellil et Rousseau}

\subsection{Opérateurs sur $\mathcal{S}_{0} \cup S_{2}$}

Soit $A$ un appartement, $x$ un sommet de $\mathcal{S}_{i} \cap A, i \in\{0,2\}$ et $C$ une chambre de $A$ contenant $x$, il existe un unique système de coordonnées sur l'appartement $A$ tel que $x=x_{0,0}, \mathcal{S} \cap C=\left\{x_{0,0}, x_{\frac{1}{2}, 0}, x_{0, \frac{1}{2}}\right\}, \tau\left(x_{\frac{1}{2}, 0}\right)=2-i$ et $\tau\left(x_{0, \frac{1}{2}}\right)=1$. Alors $\left\{x_{j, k} / j, k \in\left[0,+\infty[\}\right.\right.$ est le quartier $Q_{x, C}$ de $A$ de sommet $x$ contenant $C$. On note $V_{j, k}(x)$ l'ensemble des points $x_{j, k}$ associés aux différents choix de $A$ et $C$ tels que $x \in C \subset A$. L'ensemble $\mathcal{S}$ (resp. $\left.\mathcal{S}_{i}, \mathcal{S}_{2-i}\right)$ est réunion disjointe des $V_{j, k}(x)$ avec $(2 j, 2 k) \in \mathbb{N}^{2}$ (resp. $(j, k) \in \mathbb{N}^{2},(j-$ $\left.\left.\frac{1}{2}, k\right) \in \mathbb{N}^{2}\right)$. En fait, si $G$ est un groupe très fortement transitif les $V_{j, k}(x)$ sont les orbites du fixateur $G_{x}$ de $x$ dans $G$.

On définit des opérateurs $K_{j, k}^{i} \operatorname{sur}\left(\mathcal{S}_{0} \cup S_{2}\right)^{2}$ (pour $i \in\{0,2\}$ et $2 j, k \in \mathbb{N}$ ) par :

$$
K_{j, k}^{i}(u, v)=\left\{\begin{array}{ccc}
1 & \text { si } & \tau(u)=i \text { et } v \in V_{j, k}(u) \\
0 & \text { sinon }
\end{array}\right.
$$

On note $\mathcal{O}_{0,2}$ l'algèbre des opérateurs sur $\mathcal{S}_{0} \cup S_{2}$ qui sont invariants par un groupe $G$ très fortement transitif, conservant les types. Ainsi l'algèbre $\mathcal{O}_{0,2}$ admet pour base les $K_{j, k}^{0}$ et $K_{j, k}^{2}$, avec $j, k \in \mathbb{N}$ ou $j-\frac{1}{2}, k \in \mathbb{N}$. On peut la définir même en l'absence d'un tel groupe $G$.

Remarque 3.1: On cherche à généraliser la proposition 1.1 à cette nouvelle situation. En particulier on veut que le terme dominant pour certains produits $K_{j, k}^{i} K_{j^{\prime}, k^{\prime}}^{i^{\prime}}$ comporte un seul elément de la base ci-dessus. Pour cela on modifie la définition du degré total $N$ de l'opérateur $\sum C_{j, k}^{i} K_{j, k}^{i}$ ( non nul mais avec des constantes $C_{j, k}^{i}$ presque toutes nulles ). Une solution est de poser $N=\max \left\{3 j+2 k / C_{j, k}^{i} \neq 0\right\}$. Le terme dominant de l'opérateur est alors $\sum_{3 j+2 k=N} C_{j, k}^{i} K_{j, k}^{i}$.

Avec cette définition, un simple calcul montre que le terme dominant dans :

$$
\begin{aligned}
& K_{j, k}^{i} K_{\frac{1}{2}, 0}^{i} \text { est } K_{j+\frac{1}{2}, k}^{i}, \text { pour } j, k \in \mathbb{N} . \\
& K_{j, k}^{0} K_{\frac{1}{2}, 0}^{2},\left(\text { resp. } K_{j, k}^{2} K_{\frac{1}{2}, 0}^{0}\right), \text { pour } j-\frac{1}{2}, k \in \mathbb{N} \text { est } K_{j+\frac{1}{2}, k}^{0},\left(\text { resp. } K_{j+\frac{1}{2}, k}^{2}\right) . \\
& K_{j, k}^{i} K_{0,1}^{i}, \text { pour } j, k \in \mathbb{N} \text { est } K_{j, k+1}^{i} \cdot \\
& K_{j, k}^{0} K_{0,1}^{2},\left(\text { resp. } K_{j, k}^{2} K_{0,1}^{0}\right), \text { pour } j-\frac{1}{2}, k \in \mathbb{N} \text { est } K_{j, k+1}^{0},\left(\text { resp. } K_{j, k+1}^{2}\right) .
\end{aligned}
$$

\section{Proposition 3.2:}

1) $K_{j, k}^{i} K_{m, n}^{l}$ est non nul si et seulement si $(j, k \in \mathbb{N}$ et $i=l$ ) ou $\left(j-\frac{1}{2}, k \in \mathbb{N}\right.$ et $\left.i \neq l\right)$. 
2) L'algèbre $\mathcal{O}_{0,2}$ est non commutative et engendrée par:

$$
K_{0,0}^{0}, K_{0,0}^{2}, K_{0,1}^{0}, K_{0,1}^{2}, K_{\frac{1}{2}, 0}^{0} \text { et } K_{\frac{1}{2}, 0}^{2} .
$$

3) Pour $i, l \in\{0,2\}$ et dans le cas où $K_{j, k}^{i} K_{m, n}^{l}$ est non nul, il a pour terme dominant $K_{j+m, k+n}^{i}$.

Démonstration : La première assertion est claire.

Pour $j, k \in \mathbb{N}$ et $m+\frac{1}{2}, n \in \mathbb{N}$ on a $K_{j, k}^{i} K_{m, n}^{i} \neq 0$ alors que $K_{m, n}^{i} K_{j, k}^{i}=0$. Ainsi l'algèbre $\mathcal{O}_{0,2}$ est non commutative.

D'après la remarque 3.1 et en utilisant un raisonnement par récurrence on obtient 2). Pour la troisième assertion, par récurrence on montre que, pour $i, l \in$ $\{0,2\}$ et lorsque le produit est non nul, le terme dominant de $\left(K_{\frac{1}{2}, 0}^{i} K_{\frac{1}{2}, 0}^{l}\right)^{m}$ est $K_{m, 0}^{i}$, celui de $\left(K_{0,1}^{i}\right)^{m}$ est $K_{0, m}^{i}$, celui de $\left(K_{\frac{1}{2}, 0}^{i} K_{\frac{1}{2}, 0}^{l}\right)^{m}\left(K_{0,1}^{i}\right)^{n}$ est $K_{m, n}^{i}$ et celui de $K_{\frac{1}{2}, 0}^{i}\left(K_{\frac{1}{2}, 0}^{l} K_{\frac{1}{2}, 0}^{i}\right)^{m}\left(K_{0,1}^{l}\right)^{n}=\left(K_{\frac{1}{2}, 0}^{i} K_{\frac{1}{2}, 0}^{l}\right)^{m} K_{\frac{1}{2}, 0}^{i}\left(K_{0,1}^{l}\right)^{n}=K_{m+\frac{1}{2}, n}^{i}$. Le résultat 3) se déduit donc aussitôt par récurrence de la remarque 3.1.

Remarques 3.3: Notons $M_{0}=K_{\frac{1}{2}, 0}^{0}, M_{2}=K_{\frac{1}{2}, 0}^{2}, Y_{0}=K_{0,1}^{0}, Y_{2}=K_{0,1}^{2}$, $X_{0}=K_{\frac{1}{2}, 0}^{0} K_{\frac{1}{2}, 0}^{2}, X_{2}=K_{\frac{1}{2}, 0}^{2} K_{\frac{1}{2}, 0}^{0}, L_{1}=M_{0}+M_{2}\left(\right.$ donc $L_{1}^{2}=X_{0}+$ $\left.X_{2}\right)$ et $L_{2}=Y_{0}+Y_{2}$.

1) On a alors :

$$
\begin{aligned}
& L_{1} L_{2}=K_{\frac{1}{2}, 1}^{0}+K_{\frac{1}{2}, 1}^{2}+(q+1)\left[r K_{\frac{1}{2}, 0}^{0}+p K_{\frac{1}{2}, 0}^{2}\right] \\
& L_{2} L_{1}=K_{\frac{1}{2}, 1}^{0}+K_{\frac{1}{2}, 1}^{2}+(q+1)\left[r K_{\frac{1}{2}, 0}^{2}+p K_{\frac{1}{2}, 0}^{0}\right]
\end{aligned}
$$

Ainsi $L_{1} L_{2}=L_{2} L_{1} \Leftrightarrow p=r$.

Pour $p=r$, les opérateurs $L_{1}$ et $L_{2}$ sont ( à une constante multiplicative près) les opérateurs de Laplace considérés par Mantero et Zappa $c f$. [MZ $02 ; \S 4$ ], ils engendrent l'algèbre $\mathcal{O}_{0,2}^{*}$ des opérateurs sur $\mathcal{S}_{0} \cup \mathcal{S}_{2}$ invariants par un groupe très fortement transitif, stabilisant $\mathcal{S}_{1}$ et transitif sur $\mathcal{S}_{0} \cup$ $\mathcal{S}_{2}$. D'autre part A. M. Mantero et A. Zappa montrent dans ce cas que la transformation de Poisson est toujours bijective ( pour au moins un choix des paramètres de cette transformation ).

2) L'algèbre $\mathcal{O}_{0}=\mathbb{C}\left[X_{0}, Y_{0}\right]$ (resp. $\mathcal{O}_{2}=\mathbb{C}\left[X_{2}, Y_{2}\right]$ ) est formée des opérateurs sur $\mathcal{S}_{0}$ (resp. $\mathcal{S}_{2}$ ) invariants par un groupe $G$ très fortement transitif, conservant les types; c'est une algèbre de polynômes $c f$. [MZ 02; §3]. 


\section{Kellil et Rousseau}

L'algèbre produit $\widetilde{\mathcal{O}_{0,2}}=\mathcal{O}_{0} \times \mathcal{O}_{2}$ est formée des opérateurs de $\mathcal{O}_{0,2}$ stabilisant $\mathcal{S}_{0}$ et $\mathcal{S}_{2}$. La structure complète de l'algèbre $\mathcal{O}_{0,2}$ est donnée dans la proposition suivante :

Proposition 3.4: $\mathcal{O}_{0,2}=\mathbb{C}\left[X_{0}, Y_{0}\right] \oplus \mathbb{C}\left[X_{2}, Y_{2}\right] \oplus \mathbb{C}\left[X_{0}, Y_{0}\right] M_{0} \oplus \mathbb{C}\left[X_{2}, Y_{2}\right] M_{2}$. La structure multiplicative est donnée par les relations :

$$
\begin{aligned}
& M_{0} \cdot M_{2}=X_{0}, \quad M_{2} \cdot M_{0}=X_{2}, \quad M_{0} \cdot X_{2}=X_{0} \cdot M_{0}, \quad M_{2} \cdot X_{0}=X_{2} \cdot M_{2}, \\
& M_{0} \cdot Y_{2}=Y_{0} \cdot M_{0}+(q+1)(r-p) M_{0}, M_{2} \cdot Y_{0}=Y_{2} \cdot M_{2}+(q+1)(p-r) M_{2}, \\
& M_{i}^{2}=X_{i} \cdot M_{j}=Y_{i} \cdot M_{j}=M_{j} \cdot X_{2-i}=M_{j} \cdot Y_{2-i}=0 \text { pour } i, j \in\{0,2\}, i \neq j .
\end{aligned}
$$

Démonstration : Les relations ci-dessus sont faciles à vérifier. D'après la proposition 3.2 les monômes $X_{0}^{l} \cdot Y_{0}^{m}, X_{2}^{l} \cdot Y_{2}^{m}, X_{0}^{l} \cdot Y_{0}^{m} M_{0}$ et $X_{2}^{l} \cdot Y_{2}^{m} M_{2}$, ( pour $l, m \in \mathbb{N}$ ) sont libres. Les relations précédentes montrent que l'espace qu'ils engendrent est stable par multiplication; d'où le résultat.

\subsection{Opérateurs sur $\mathcal{S}_{1}$}

Pour $u, v \in \mathcal{S}_{1}$ et $k, l$ tels que $2 k, 2 l$ et $k+l \in \mathbb{N}$. On dit que $v \in V_{k, l}^{*}(u)$ si $u$ et $v$ sont dans un même appartement $A$ et s'il existe dans cet appartement un système orthogonal de coordonnées tel que $x_{0,0}=u, x_{k, l}=v$ et les sommets de type 0 (resp. 2,1) s'ecrivent $x_{i, j}$ avec $\left(i, j+\frac{1}{2}\right) \in \mathbb{Z}^{2}$ (resp. $\left(i+\frac{1}{2}, j\right) \in$ $\left.\mathbb{Z}^{2},(2 i, 2 j, i+j) \in \mathbb{Z}^{3}\right)$.

Avec cette définition, si $G$ est très fortement transitif et conserve les types, alors, pour $g \in G$ et $u, v \in \mathcal{S}_{1}$, on a : $g v \in V_{k, l}^{*}(g u) \Leftrightarrow v \in V_{k, l}^{*}(u)$. L'ensemble $\mathcal{S}_{1}$ est réunion disjointe des $V_{k, l}^{*}(u)$ avec $2 k, 2 l$ et $k+l \in \mathbb{N}$ qui sont les orbites dans $\mathcal{S}_{1}$ du fixateur $G_{u}$ de $u$ dans $G$.

Pour $k, l$ ainsi choisis, on définit des opérateurs $K_{k, l}^{*}$ sur $\mathcal{S}_{1} \times \mathcal{S}_{1}$ par :

$$
K_{k, l}^{*}(u, v)=\left\{\begin{array}{ccc}
1 & \text { si } & v \in V_{k, l}^{*}(u) \\
0 & \text { sinon }
\end{array}\right.
$$

On vérifie facilement que l'algèbre $\mathcal{O}_{1}$ des opérateurs sur $\mathcal{S}_{1} \times \mathcal{S}_{1}$, invariants par un groupe $G$ très fortement transitif et respectant les types admet comme base les $K_{k, l}^{*}$ avec $2 k, 2 l \in \mathbb{N}$ et $k+l \in \mathbb{N}$. On peut ainsi la définir même en l'absence d'un tel groupe $G$.

Proposition 3.5: L'algèbre $\mathcal{O}_{1}$ est non commutative. Elle est engendrée par

$$
K_{0,0}^{*}, K_{\frac{1}{2}, \frac{1}{2}}^{*}, K_{1,0}^{*} \quad \text { et } K_{0,1}^{*}
$$


Démonstration : On appelle terme dominant de l'opérateur $\sum C_{k, l}^{*} K_{k, l}^{*}$ ( non nul mais avec des constantes $C_{k, l}^{*}$ presque toutes nulles ) l'opérateur $\sum_{k+l=N} C_{k, l}^{*} K_{k, l}^{*}$ où $N=\max \left\{k+l / C_{k, l}^{*} \neq 0\right\}$ est le degré total.

On voit facilement que le terme dominant de $K_{1,0}^{*} K_{\frac{1}{2}, \frac{1}{2}}^{*}\left(\right.$ resp. $\left.K_{\frac{1}{2}, \frac{1}{2}}^{*} K_{1,0}^{*}\right)$ est $K_{\frac{3}{2}, \frac{1}{2}}^{*}\left(\right.$ resp. $\left.K_{\frac{1}{2}, \frac{3}{2}}^{*}\right)$, ainsi l'algèbre $\mathcal{O}_{1}$ n'est pas commutative. Notons $\mathcal{O}_{1}^{\prime}$ l'algèbre engendrée par $K_{0,0}^{*}, K_{\frac{1}{2}, \frac{1}{2}}^{*}, K_{1,0}^{*}$ et $K_{0,1}^{*}$. On remarque par ailleurs que pour $i$ (resp. $j) \in \mathbb{N}^{*}$ le terme dominant de $K_{i, 0}^{*} K_{1,0}^{*}\left(\operatorname{resp} . K_{0, j}^{*} K_{0,1}^{*}\right)$ est $K_{i+1,0}^{*}\left(\right.$ resp. $\left.K_{0, j+1}^{*}\right)$. Par un raisonnement par récurrence on voit que $\mathcal{O}_{1}^{\prime}$ contient $K_{i, 0}^{*}$ pour $i \in \mathbb{N}^{*}$ et $K_{0, j}^{*}$ pour $j \in \mathbb{N}^{*}$. Puis en utilisant que le terme dominant de $K_{i, j}^{*} K_{\frac{1}{2}, \frac{1}{2}}^{*}$ est $K_{i+\frac{1}{2}, j+\frac{1}{2}}^{*}$ pour $i+j \in \mathbb{N}$ et par récurrence on obtient dans $\mathcal{O}_{1}^{\prime}$ tous les autres termes $K_{i, j}^{*}$. Ainsi $\mathcal{O}_{1}=\mathcal{O}_{1}^{\prime}$.

Remarques 3.6: Posons $L_{1}=K_{\frac{1}{2}, \frac{1}{2}}^{*}$ et $L_{2}=K_{1,0}^{*}+K_{0,1}^{*}$. On a alors : $L_{1} L_{2}=L_{2} L_{1}=K_{\frac{1}{2}, \frac{3}{2}}^{*}+K_{\frac{3}{2}, \frac{1}{2}}^{*}+q(p+r) L_{1}+(q-1)\left[(p+1) K_{1,0}^{*}+(r+1) K_{0,1}^{*}\right]$.

1) L'algèbre engendrée par $L_{1}$ et $L_{2}$ est l'algèbre $\mathbb{C}\left[L_{1}, L_{2}\right]$ des polynômes en $L_{1}$ et $L_{2}$. En effet le monôme $L_{2}^{m} L_{1}^{n}$ est égal à $K_{m+\frac{n}{2}, \frac{n}{2}}^{*}$ plus une combinaison linéaire des $K_{i, j}^{*}$ avec $(i+j, i)<\left(m+\frac{n}{2}, \frac{n}{2}\right)$ pour l'ordre lexicographique. Ainsi $\left\{L_{2}^{m} L_{1}^{n}\right\}$ forme une famille libre.

2) Si $p=r$, l'algèbre $\mathcal{O}_{1}^{*}$ des opérateurs sur $\mathcal{S}_{1}$ invariants par un groupe $G$ très fortement transitif, transitif sur $\mathcal{S}_{0} \cup \mathcal{S}_{2}$ admet pour base les $K_{k, l}^{*}+K_{l, k}^{*}$ ( pour $2 k, 2 l, k+l \in \mathbb{N}$ ). C'est aussi l'algèbre $\mathbb{C}\left[L_{1}, L_{2}\right]$ des polynômes en $L_{1}$ et $L_{2}, c f$. [MZ 02; $\left.\S 5\right]$. Dans certains cas $\left(q=p\right.$ ou $\left.q=p^{2}\right)$, A. M. Mantero et A. Zappa montrent qu'il existe des fonctions propres de $\mathcal{O}_{1}^{*}$ qui ne sont pas une transformation de Poisson d'une mesure additive finie sur $\Omega$.

3) Notons $L_{2}^{-}=K_{0,1}^{*}-K_{1,0}^{*}$, on a alors $K_{0,1}^{*}=\frac{1}{2}\left[L_{2}+L_{2}^{-}\right], \quad K_{1,0}^{*}=$ $\frac{1}{2}\left[L_{2}-L_{2}^{-}\right]$et la structure de l'algèbre $\mathcal{O}_{1}$ est donnée par la proposition suivante :

Proposition 3.7: $\mathcal{O}_{1}=\mathbb{C}\left[L_{1}, L_{2}\right] \oplus \mathbb{C}\left[L_{1}, L_{2}\right] L_{2}^{-}$, avec les relations :

$$
\begin{aligned}
L_{2}^{-} L_{1}= & -L_{1} L_{2}^{-}+2 q(r-p) L_{1}+(q-1)\left[(r-p) L_{2}+(r+p+2) L_{2}^{-}\right] \\
L_{2}^{-} L_{2}= & L_{2} L_{2}^{-}-2(q-1) L_{1} L_{2}^{-}+2 q(q-1)(r-p) L_{1}+(q-1)^{2}\left[(r-p) L_{2}+\right. \\
& \left.+(r+p+2) L_{2}^{-}\right] \\
\left(L_{2}^{-}\right)^{2}= & L_{2}^{2}-4 q L_{1}^{2}-2(q-1) L_{1}\left[L_{2}-q(p+r)\right]+\left(q^{2}+1\right)\left[(r+p+2) L_{2}+\right. \\
& \left.(r-p) L_{2}^{-}\right]+4 q^{2}(r+1)(p+1) I d .
\end{aligned}
$$




\section{Kellil et Rousseau}

Démonstration : Les relations ci-dessus sont des conséquences faciles des relations élémentaires suivantes :

$$
\begin{aligned}
& \left(K_{\frac{1}{2}, \frac{1}{2}}^{*}\right)^{2}=K_{1,1}^{*}+(p+1) K_{1,0}^{*}+(r+1) K_{0,1}^{*}+q(r+1)(p+1) K_{0,0}^{*} \\
& L_{1} K_{1,0}^{*}=K_{\frac{1}{2}, \frac{3}{2}}^{*}+p q L_{1}+(p+1)(q-1) K_{1,0}^{*} \\
& L_{1} K_{0,1}^{*}=K_{\frac{3}{2}, \frac{1}{2}}^{*}+r q L_{1}+(r+1)(q-1) K_{0,1}^{*} \\
& K_{1,0}^{*} L_{1}=K_{\frac{3}{2}, \frac{1}{2}}^{*}+p q L_{1}+(p+1)(q-1) K_{1,0}^{*} \\
& K_{0,1}^{*} L_{1}=K_{\frac{1}{2}, \frac{3}{2}}^{*}+r q L_{1}+(r+1)(q-1) K_{0,1}^{*} \\
& K_{1,0}^{*} K_{0,1}^{*}=q K_{1,1}^{*}+(q-1) K_{\frac{3}{2}, \frac{1}{2}}^{*} \\
& K_{0,1}^{*} K_{1,0}^{*}=q K_{1,1}^{*}+(q-1) K_{\frac{1}{2}, \frac{3}{2}}^{*} .
\end{aligned}
$$

Ainsi l'espace vectoriel engendré par les $L_{2}^{m} L_{1}^{n}$ et $L_{2}^{m} L_{1}^{n} L_{2}^{-}$(pour $m$ et $n \in \mathbb{N}$ ) est bien égal à $\mathcal{O}_{1}$. On va voir que ces monômes en forment une base.

On vérifie facilement que le terme dominant de :

$$
K_{i, j}^{*} K_{\frac{1}{2}, \frac{3}{2}}^{*}\left(\text { resp. } K_{i, j}^{*} K_{\frac{3}{2}, \frac{1}{2}}^{*}\right) \text { est } \begin{cases}K_{i+\frac{1}{2}, j+\frac{3}{2}}^{*}\left(\operatorname{resp} . K_{i+\frac{3}{2}, j+\frac{1}{2}}^{*}\right. & \text { si } i, j \in \mathbb{N} \\ K_{i+\frac{3}{2}, j+\frac{1}{2}}^{*}\left(\operatorname{resp} . K_{i+\frac{1}{2}, j+\frac{3}{2}}^{*}\right) & \text { si } i, j \notin \mathbb{N}\end{cases}
$$

auquel on doit ajouter $K_{i+1, j+1}^{*}$ affecté d'un certain coefficient. Il est alors facile de montrer que le degré total de $Q\left[K_{\frac{1}{2}, \frac{3}{2}}^{*}-K_{\frac{3}{2}, \frac{1}{2}}^{*}\right]$ pour $Q \in \mathcal{O}_{1}$ est égal à $2+$ le degré total de $Q$. En particulier $K_{\frac{1}{2}, \frac{3}{2}}^{\frac{1}{2}, \frac{3}{2}}-K_{\frac{3}{2}, \frac{1}{2}}^{*}$ n'est pas un diviseur de 0 (à droite). Or une relation linéaire entre les monômes $L_{2}^{m} L_{1}^{n}$ et $L_{2}^{m} L_{1}^{n} L_{2}^{-}$ s'écrit $P(L)=Q(L) L_{2}^{-}$où $P$ et $Q$ sont des polynômes en $L_{1}$ et $L_{2}$. On a alors :

$0=P(L) L_{1}-L_{1} P(L)=Q(L)\left(L_{2}^{-} L_{1}-L_{1} L_{2}^{-}\right)=2 Q(L)\left(K_{\frac{1}{2}, \frac{3}{2}}^{*}-K_{\frac{3}{2}, \frac{1}{2}}^{*}\right)$, ce qui impose $Q(L)=0$, donc $P(L)=0$. D'où le résultat.

\subsection{Fonctions propres radiales}

Des calculs assez faciles analogues à ceux du $\S 2$ montrent que les espaces propres de $\mathcal{O}_{0,2}$ (resp. $\mathcal{O}_{1}$ ) dans l'espace des fonctions s-radiales par rapport à un sommet $s$ de type 0 ou 2 (resp. 1) sont de dimension au plus 1 ; ceci ne permet pas d'obtenir de résultat concernant la conjecture d'Helgason avec les méthodes du $\S 2$. 


\section{Commentaires}

Ichiro Satake [S 63] a étudié les groupes réductifs sur un corps p-adique. Si $G$ est un tel groupe et $U$ un "bon" sous-groupe compact ouvert, alors l'algèbre de Hecke, $\mathcal{H}(G, U)$, c'est à dire l'algèbre de convolution des fonctions complexes bi- $U$-invariantes à support compact, est toujours commutative si $G$ est connexe. Elle est même intègre de degré de transcendance le rang relatif de $G$, et on sait déterminer des générateurs explicites.

A un tel groupe $G$ est associé son immeuble de Bruhat-Tits $\Delta$ que l'on va supposer ici de type $\widetilde{A_{2}}$ ou $\widetilde{B_{2}}$. Alors $G$ est un groupe très fortement transitif sur $\Delta$. Pour l'action sur les types on obtient tous les cas de figure envisagés dans les paragraphes précédents. Si le groupe $G$ est simplement connexe il conserve les types. Si le groupe $G$ est deployé de type $A_{2}\left(\right.$ resp. $\left.B_{2}\right)$ et adjoint il permute circulairement les types ( resp. échange les types 0 et 2 ) des sommets de l'immeuble $\Delta$ qui est de type $\widetilde{A_{2}}\left(\right.$ resp. $\left.\widetilde{B_{2}}\right)$. Dans le cas $\widetilde{A_{2}}$ pour obtenir un groupe $G$ échangeant les types 1 et 2 , il faut remplacer $G$ par un groupe non connexe faisant intervenir un automorphisme exterieur ( par exemple un produit semi-direct).

Pour faire le lien entre notre point de vue et celui de Satake il faut choisir pour $U$ le fixateur $K_{s}$ dans $G$ d'un sommet $s$ de type $i$. Alors $G / U$ s'identifie à l'ensemble $\mathcal{S}_{G i}$ des sommets de type appartenant à l'orbite $G i$ de $i$ sous $G$. Ainsi $\mathcal{H}(G, U)$ s'identifie à l'algèbre $\mathcal{O}_{G i}$ des opérateurs $G$-invariants sur $\mathcal{S}_{G i}$.

Examinons de plus près les conditions d'application du théorème de Satake. Il faut d'abord que $G$ soit connexe, ce qui n'est pas le cas pour $\mathcal{O}_{1,2}$ examiné en 1.3 , on a d'ailleurs trouvé dans ce cas que $\mathcal{O}_{1,2}$ est non commutative. Il faut ensuite que $U=K_{s}$ soit un "bon" sous-groupe compact ouvert ce qui se traduit par les conditions techniques I et II de Satake. La condition II est essentiellement conséquence des proprietés connues des immeubles ( car on a choisi $U=K_{s}$ ). La condition I se résume en l'existence de décompositions dans $G: G=U . H . N$ ( Iwasawa ) et $G=U . H . U$ ( Bruhat ). Cette décomposition de Bruhat est vérifiée si et seulement si $U=K_{s}$ induit tout le groupe de Weyl relatif sur un appartement contenant $s$; cela élimine donc le cas de l'algèbre $\mathcal{O}_{1}$ pour $\Delta$ de type $\widetilde{B_{2}}$ ( on a bien trouvé dans la sous-section 3.2 que $\mathcal{O}_{1}$ est non commutative). La décomposition d'Iwasawa est vérifiée essentiellement si $U=K_{s}$ est transitif sur $\Omega$, ce qui conduit à la même élimination.

Pour les autres cas envisagés ici, $\mathcal{O}_{\text {circ }}$ et $\mathcal{O}_{i}(c f . \S 1)$ vérifient les hy- 


\section{KeLlil et Rousseau}

pothèses de Satake avec un groupe de type $A_{2}$ adjoint ou simplement connexe. Pour $\widetilde{B_{2}}$ et si $G$ respecte les types, $\mathcal{S}_{0} \cup \mathcal{S}_{2}$ n'est pas une orbite de $G$ et la non commutativité de $\mathcal{O}_{0,2}$ ne contredit pas Satake. Pour $\widetilde{B_{2}}$ et $G$ très fortement transitif échangeant les types 0,2 on a vu que les algèbres $\mathcal{O}_{0,2}^{*}$ et $\mathcal{O}_{1}^{*}$ sont bien commutatives.

\section{Références}

[C 01] D.I. Cartwright. Spherical harmonic analysis on buildings of type $\widetilde{A_{n}}$. Monatsh. Math. 133, 93-109 (2001).

[CM 94] D.I. Cartwright and W. Mlotkowski. Harmonic analysis for groups acting on triangle buildings. J. Austral. Math. Soc. (Series A). 56, 345-383 (1994).

[CW 04] D.I. Cartwright, W. Woess. Isotropic random walks in a building of type $\widetilde{A_{d}}$. Math. Z. 247, 101-135 (2004).

[GL 99] P. Gerardin et K.F. Lai. Opérateurs invariants sur les immeubles affines de type A. C. R. Acad. Sci. Paris ser. I, 329, 1-4 (1999).

[McD 71] I.G. Mac Donald. Spherical functions on a group of p-adic type. Ramanujan Inst. Publ. 2 Université de Madras (1971).

[MZ 00] A. M. Mantero et A. Zappa. Eigenfunctions of the Laplace operators for a building of type $\widetilde{A_{2}}$. J. Geom. Analysis. 10, 339-363 (2000).

[MZ 02] A. M. Mantero et A. Zappa. Eigenfunctions of the Laplace operators for buildings of type $\widetilde{B_{2}}$. Boll. Unione Mat. Ital. Ser B Artic. Ric. Mat. (8)5, 163-195 (2002).

[R 89] M. Ronan. Lectures on buildings, Perspect. in Math. 7. Acad. Press. New York (1989).

[S 63] I. Satake. Theory of spherical functions on reductive algebraic groups over p-adic fields. Pub. Math. I. H. E. S, 18, 5-69 (1963).

Ferdaous Kellil

FACUlté DES SCIEnCES DE MonastiR

DÉpartement de Mathématiques

5000 Monastir

TUNisie

kellilferdaous@yahoo.fr

\section{Guy Rousseau}

Institut Elie Cartan, UMR 7502

NANCY-Université, CNRS, INRIA

BP 239

54506 Vandoeuvre Les Nancy

FRANCE

Guy.Rousseau@iecn.u-nancy.fr 